\title{
Title: Adaptive Composite Overlapping Mesh Algorithms on Message Passing Architectures
}

\author{
Author(s): D. L. Brown, CIC-19 \\ D. J. Quinlan, CIC-19 \\ G. S. Chesshire, CIC-19 \\ W. D. Henshaw, CIC-19 \\ M. Berndt, Dept. Appl. Math., U. Colorado \\ Submitted to:

\section{DISCLAIMER}

This report was prepared as an account of work sponsored by an agency of the United States Government. Neither the United States Government nor any agency thereof, nor any of their employees, makes any warranty, express or implied, or assumes any legal liability or responsibility for the accuracy, completeness, or usefulness of any information, apparatus, product, or process disclosed, or represents that its use would not infringe privately owned rights. Reference herein to any specific commercial product, process, or service by trade name, trademark, manufacturer, or otherwise does not necessarily constitute or imply its endorsement, recommendation, or favoring by the United States Government or any agency thereof. The views and opinions of authors expressed herein do not necessarily state or reflect those of the United States Government or any agency thereof.

\section{Los Alamos}

Los Alamos National Laboratory, an aftirmative action/equal opportunity employer, is operated by the University of California for the U.S. Department of Energy under contract W-7405-ENG-36. By acceptance of this article, the publisher recognizes that the U.S. Government retains a nonexclusive, royaltyfree license to publish or reproduce the published form of this contribution, or to allow others to do so, for U.S. Government purposes. Los Alamos National Laboratory requests that the publisher identify this article as work performed under the auspices of the U.S. Department of Energy. Los Alamos National Laboratory strongly supports academic treedom and a researcher's right to publish; as an institution, however, the Laboratory does not endorse the viewpoint of a publication or guarantee its technical correctness. 


\section{DISCLAMMER}

Portions of this document may be illegible in electronic image products. Images are produced from the best available original document. 


\title{
Adaptive Composite Overlapping Mesh Algorithms on Message Passing Architectures
}

\author{
David L. Brown, ${ }^{1}$ Daniel J. Quinlan, Geoffrey S, Chesshire, and William D. Henshaw \\ Computing, Information, and Communications Division, Los Alamos National Laboratory \\ Markus Berndt \\ Department of Applied Mathematics, University of Colorado
}

\begin{abstract}
This is the final report of a three-year, Laboratory Directed Research and Development (LDRD) project at the Los Alamos National Laboratory (LANL). The adaptive composite overlapping mesh method is a flexible technique for representing complex geometry and obtaining high computational resolution in the context of finite-difference and finite-volume computations of various fluid flow equations. This project developed high-level computational tools that facilitate the development of fluid flow simulation software using this method. An object-oriented approach was taken which used the $\mathrm{C}++$ programming language to develop libraries containing $\mathrm{C}++$ classes that provide abstractions of the basic operations required to assemble the required simulation software. Through this object-oriented approach, it was also possible to hide many of the details of the computations required on parallel computer architectures. The result of this research is the basis for a high-level programming environment that facilitates the development of high-resolution simulation software on serial or parallel computer architectures for the equations describing fluid flow in regions with complex geometry.
\end{abstract}

\section{Background and Research Ubjectives}

The advent of high-performance serial and parallel computers has opened the possibility of performing high-resolution simulations of fluid flow phenomena in regions with complex geometry. The method of composite overlapping grids [1,2] provides a mechanism for accurately representing complex geometry by using a set of overlapping structured meshes on which finite-difference or finite-volume methods can easily be used to approximate the partial differential equations. The method of block-structured adaptive mesh refinement (AMR) [3] has been extended to this environment as well, and provides a mechanism for automatically obtaining high computational resolution while only refining the mesh in regions where additional refinement is needed. While the potential of these methods is great, the rapid development of simulation software that incorporates both overlapping grids and AMR is made difficult both by the complex data structures that are involved in each of the methods, and by the complexities of programming for modern massively parallel computer architectures. The objective of this research project was to initiate the development of programming tools that

${ }^{1}$ Principal Investigator, E-mail: dlb@lanl.gov 
would facilitate such software development by using object-oriented techniques to hide much of the complexity at the highest programming levels. The $\mathrm{C}++$ programming language provided much of the necessary capability required to accomplish this task. $\mathrm{C}++$ classes provided a natural mechanism for accomplishing these objectives. Using the full power of the $\mathrm{C}++$ language, classes were developed for handling the details of parallel array operations and for encapsulating the details of objects such as grids, grid functions and differential operators.

\section{Importance to LANL's Science and Technology Base and National R\&D Needs}

One of the Laboratory's key areas of expertise is the simulation of fluid phenomena using supercomputers. The past Laboratory emphasis on fluid behavior of programmatic interest in simple geometries has been expanded by the more recent interest in promoting technology transfer between the national laboratories and U.S. industry. For example, fluidsdriven processes requiring high computational resolution, and often involving complicated geometries, are of interest to industrial scientists modeling internal combustion, materials processing and manufacture, as well as many other applications of critical industrial technology. Another trend is the evolution of supercomputer technology towards massively parallel processors. Parallel machines have few language standards, which makes code portability difficult. In addition, algorithmic complexity is increasing in proportion to the ambitiousness of the applications that scientists hope to simulate. The development of computational tools that simplify the development of simulation software that addresses these application targets and that is also portable across the full range of modern computer architectures is of critical interest to both the Laboratory and to the nation.

\section{Scientific Approach and Accomplishments}

The objective of developing high-level computational tools that simplify the computational scientist's job of writing software capable of simulating fluid flows in complex geometries has been accomplished by using an object-oriented approach based on the $\mathrm{C}++$ programming language. In the object-oriented approach, basic computational objects are abstracted, and classes are developed that encapsulate the data structures and functionality of each object. The use of the $\mathrm{C}++$ language helps to enforce a strictly-defined interface to each object, which in turn allows the objects to be developed independently of each other. The algorithms used to implement each object can be changed to improve performance or functionality, for example, without affecting the object's interface. In addition, different architecture-dependent implementations of objects can be supported with access through only one well-defined interface, allowing the development of high-level code to be essentially architecture independent. Since the fluid flow simulation codes are developed at a high level, 
they access the data and functionality of these objects only through their well-defined interfaces, and thus do not have to be rewritten every time that the implementation of the objects changes. This results in high-level software that is easily written and maintained.

As part of this project, the $\mathrm{A}++/ \mathrm{P}++$ array classes were further developed to handle the basic array operations required in scientific codes, in particular those using the composite overlapping grid method. The P++ class was ported to both the CM-5 and T3D computers as well as to generic parallel environments that support the PVM and MPI message passing libraries [4]. In addition, the Overture Framework [5] was developed, which includes C++ libraries for handling grids [6], grid functions [7] and differential operators [8] on composite overlapping grids. This Framework provides a very powerful tool that allows the development of fluids simulation software that appears to be independent of the complex grid data structures that it accesses and uses. The original objective of developing software libraries to support adaptive mesh refinement for overlapping grids on parallel architectures was not met due to the unanticipated complexity of this objective. However, the development of the AMR++ library, which is intended to address this need, continues as part of a spin-off of this LDRD project.

\section{References and Publications}

[1] Chesshire, G., and W. Henshaw, "Composite Overlapping Meshes for the Solution of Partial Differential Equations," Journal of Computational Physics, 90, pp. 1-64, (1990).

[2] Brown, D., G. Chesshire, W. Henshaw and H.O. Kreiss, "On Composite Overlapping Grids," Proceedings of the Seventh International Conference on Finite Element Methods in Flow Problems, Huntsville, Alabama, 1989.

[3] Brislawn, K., D. Brown, G. Chesshire and J. Saltzman, "Adaptively-Refined Overlapping Grids for the Numerical Solution of Systems of Hyperbolic Conservation Laws," Los Alamos National Laboratory document LA-UR-95-257.

[4] Quinlan, D., "A++/P++ Manual," Los Alamos National Laboratory document LA-UR95-3273, and "A++/P++ Array Class Library," Los Alamos Computer Code LA-CC-961.

[5] Brown, D., and W. Henshaw, "Overture: An Advanced Object-Oriented Software System for Moving Overlapping Grid Computations," Los Alamos National Laboratory document LA-UR-96-2931.

[6] Chesshire, G., "Overture, the Grid Classes," Los Alamos National Laboratory document LA-UR-96-3708.

[7] Henshaw, W., "Grid Functions for Overture," Los Alamos National Laboratory document LA-UR-96-3464. 
[8] Brown, D., "Classes for Finite Volume Operators and Projection Operators," Los Alamos National Laboratory document LA-UR-96-3470. 